\title{
The Topological Entropy Mechanism of Coronavirus Disease 2019 (COVID-19)
}

\author{
Ruomeng Xu1 ${ }^{1}$, Ludan Lei ${ }^{2}$, Ruihua Qin ${ }^{3}$, Zhende Huang ${ }^{3}$, Kuo-Chen Chou ${ }^{4}$ \\ ${ }^{1}$ Brimmer and May School, Chestnut, MA, USA; ${ }^{2}$ Southwest University of Finance and Economics, Chengdu, China; \\ ${ }^{3}$ School of Biomedical Engineering, Shanghai Jiao Tong University, Shanghai, China; ${ }^{4}$ Gordonlife Science Institute, \\ Boston. MA, USA
}

Correspondence to: Ruomeng Xu, mxu@brimmer.org; Ludan Lei, lldo521@163.com Ruihua Qin, qin16880109@sina.com; Zhende Huang, davidhuang10203@sina.com; Kuo-Chen Chou, kcchou@gordonlifescience.org, kcchou38@gmail.com

Keywords: Vaccine, Cellular, Coronavirus, Pandemic COVID-19, Spatial and Time Entropy, Topological Entropy, Chou's 5-Steps Rule

Received: November 17, 2020

Accepted: December 20, 2020

Published: December 23, 2020

Copyright $\odot 2020$ by author(s) and Scientific Research Publishing Inc.

This work is licensed under the Creative Commons Attribution International License (CC BY 4.0).

http://creativecommons.org/licenses/by/4.0/

\section{(c) (i) Open Access}

\section{ABSTRACT}

The biological principal or its detailed mechanism for the pandemic coronavirus disease 2019 (COVID-19) has been investigated and analyzed from the topological entropy approach. The findings thus obtained have provided very useful clues and information for developing both powerful and safe vaccines against the pandemic COVID-19.

\section{INTRODUCTION}

Originally initiated in Italy or Span, coronavirus disease 2019 (COVID-19) is a contagious disease caused by severe acute respiratory syndrome coronavirus 2 (SARS-CoV-2).

In December 2019, the COVID-19 was also discovered in Wuhan, China. It has since spread to 220 countries worldwide. cough.

Symptoms of COVID-19 are variable, but often include fatigue, breathing difficulties, fever, and

According to the recent report, there are about 13,000,000 confirmed cases in USA alone, of which about 300,000 are confirmed to death.

Meanwhile, as clearly pointed out by the famous American news magazine TIME, the year of 2020 is the most serious disaster year for our earth. And such a statement has also been shown via its Cover Figure selection.

The virus that causes COVID-19 spreads mainly when an infected person is in close contact with another person. Small droplets and aerosols containing the virus can spread from an infected person's nose and mouth as he breathes, coughs, sneezes, sings, or speaks.

Preventive measures include social distancing, quarantining, ventilation of indoor spaces, covering 
coughs and sneezes, hand washing, and keeping unwashed hands away from the face. The use of face masks or coverings has been recommended in public settings to minimize the risk of transmissions.

Recently, four intriguing papers were published with the attempt to find the biological mechanism of COVID-19 from the mathematical [1], physical [2], chemical [3] and physiochemical [2] approaches, respectively. In this paper, we are to address such an extremely important topic from a different approach.

In mathematics, the topological entropy of a topological, dynamical system is a nonnegative, extended real number used for measuring the complexity of the system, as formulated below:

$$
\lim _{w \rightarrow \infty}\left(\lim \frac{\log R(w, t)}{t}\right)
$$

For more information, see a Wikipedia article at https://en.wikipedia.org/wiki/Topological_entropy, as well as Figure $1 \&$ Figure 2 in the Section 2.

\section{METERIALS AND METHOD}

The "Chou's 5-step rules" or "Chou's 5-steps rule" has also been applied because it has been widely and increasingly used by many scientists to study biological sequence analyses [4-52].

To develop a really useful predictor for a biological system, one needs to go through the following five steps: 1) select or construct a valid benchmark dataset to train and test the predictor; 2) represent the samples with an effective formulation that can truly reflect their intrinsic correlation with the target to be predicted; 3) introduce or develop a powerful algorithm to conduct the prediction; 4) properly perform cross-validation tests to objectively evaluate the anticipated prediction accuracy; 5) establish a user-friendly web-server for the predictor that is accessible to the public. Papers presented for developing a new sequence-analyzing method or statistical predictor by observing the guidelines of Chou's 5-step rules have the following notable merits: 1) crystal clear in logic development, 2) completely transparent in operation, 3) easily to repeat the reported results by other investigators, 4) with high potential in stimulating other sequence-analyzing methods, and 5) very convenient to be used by the majority of experimental scientists.

Accordingly, the webserver for the current paper has been established as well.

Illustration to show the 204 cellular automata

\begin{tabular}{|c|c|}
\hline $111 \rightarrow 1,110 \rightarrow>1,101 \rightarrow 0,100 \rightarrow 0$, \\
\hline $011 \rightarrow>1,010 \rightarrow>1,001 \rightarrow 0,000 \rightarrow 0$.
\end{tabular}

Figure 1. The schematic drawing for the cellular automata.

Illustration to show the 184 cellular automata

\begin{tabular}{c}
\hline $111 \rightarrow 1,110 \rightarrow 0,101 \rightarrow 1,100 \rightarrow>1$, \\
\hline $011 \rightarrow 1,010 \rightarrow 1,001 \rightarrow 0,000 \rightarrow 0$.
\end{tabular}

Figure 2. The schematic drawing for the cellular automata.

\section{CONCLUSION}

The topological entropy and the detailed mechanism therein are very useful for developing extremely powerful and high efficiency vaccines in fighting against the pandemic COVID-19 as well as saving the human beings on this earth.

\section{CONFLICTS OF INTEREST}

The authors declare no conflicts of interest regarding the publication of this paper. 


\section{REFERENCES}

1. Fang, L., Wang, X., Lai, Z., Zhang, D., Wu, M., Pan, Z., Wang, L., Tang, K., Qian, D., Huang, Z., Wang, X., Chen, H. and Chou, K.C. (2020) Reveal the Molecular Principle of Coronavirus Disease 2019 (COVID-19). Index of Sciences, 12, 1-13. https://doi.org/10.4236/ns.2020.1211063

2. Zhang, D., Fang, L., Wang, L., Pan, Z., Lai, Z., Wu, M., Tang, K., Ludan, L., Qian, D., Huang, Z., Wang, X., Chen, H. and Chou, K.C. (2020) The Physical Principle of Coronavirus Disease 2019 (COVID-19). Index of Sciences, 12, 1-13. https://doi.org/10.4236/ns.2020.1211063

3. Zhang, D., Fang, L., Wang, L., Pan, Z., Lai, Z., Wu, M., Tang, K., Ludan, L., Qian, D., Huang, Z., Wang, X., Chen, H. and Chou, K.C. (2020) The Chemical Mechanism of Pestilences or Coronavirus Disease 2019 (COVID-19). Natural Science, 12, 717-725. https://doi.org/10.4236/ns.2020.1211063

4. Butt, A.H. and Khan, Y.D. (2018) Prediction of S-Sulfenylation Sites Using Statistical Moments Based Features via Chou's 5-Step Rule. International Journal of Peptide Research and Therapeutics (IJPRT), 17, 711-738.

5. Awais, M., Hussain, W., Khan, Y.D., Rasool, N., Khan, S.A. and Chou, K.C. (2019) iPhosH-PseAAC: Identify Phosphohistidine Sites in Proteins by Blending Statistical Moments and Position Relative Features According to the Chou's 5-Step Rule and General Pseudo Amino Acid Composition. IEEE/ACM Transactions on Computational Biology and Bioinformatics, 19, 1-21. https://doi.org/10.1109/TCBB.2019.2919025

6. Barukab, O., Khan, Y.D., Khan, S.A. and Chou, K.C. (2019) iSulfoTyr-PseAAC: Identify Tyrosine Sulfation Sites by Incorporating Statistical Moments via Chou's 5-Steps Rule and Pseudo Components. Current Genomics, 20, 306-320. https://doi.org/10.2174/1389202920666190819091609

7. Butt, A.H. and Khan, Y.D. (2019) Prediction of S-Sulfenylation Sites Using Statistical Moments Based Features via Chou's 5-Step Rule. International Journal of Peptide Research and Therapeutics (IJPRT), 19, 71-82. https://doi.org/10.1007/s10989-019-09931-2

8. Chen, Y. and Fan, X. (2019) Use Chou's 5-Steps Rule to Reveal Active Compound and Mechanism of Shuangsheng Pingfei San on Idiopathic Pulmonary Fibrosis. Current Molecular Medicine, 19, 511-563.

9. Du, X., Diao, Y., Liu, H. and Li, S. (2019) MsDBP: Exploring DNA-Binding Proteins by Integrating Multi-Scale Sequence Information via Chou's 5-Steps Rule. Journal of Proteome Research, 18, 3119-3132. https://doi.org/10.1021/acs.jproteome.9b00226

10. Dutta, A., Dalmia, A., R, A., Singh, K.K. and Anand, A. (2019) Using the Chou's 5-Steps Rule to Predict Splice Junctions with Interpretable Bidirectional Long Short-Term Memory Networks. Computers in Biology and Medicine, 116, Article ID: 103558. https://doi.org/10.1016/j.compbiomed.2019.103558

11. Ehsan, A., Mahmood, M.K., Khan, Y.D., Barukab, O.M., Khan, S.A. and Chou, K.C. (2019) iHyd-PseAAC (EPSV): Identify Hydroxylation Sites in Proteins by Extracting Enhanced Position and Sequence Variant Feature via Chou's 5-Step Rule and General Pseudo Amino Acid Composition. Current Genomics, 20, 124-133. https://doi.org/10.2174/1389202920666190325162307

12. Hussain, W., Khan, S.D., Rasool, N., Khan, S.A. and Chou, K.C. (2019) SPalmitoylC-PseAAC: A Sequence-Based Model Developed via Chou's 5-Steps Rule and General PseAAC for Identifying S-Palmitoylation Sites in Proteins. Analytical Biochemistry, 568, 14-23. https://doi.org/10.1016/j.ab.2018.12.019

13. Hussain, W., Khan, Y.D., Rasool, N., Khan, S.A. and Chou, K.C. (2019) SPrenylC-PseAAC: A Sequence-Based Model Developed via Chou's 5-Steps Rule and General PseAAC for Identifying S-Prenylation Sites in Proteins. Journal of Theoretical Biology, 468, 1-11. https://doi.org/10.1016/j.jtbi.2019.02.007

14. Jun, Z. and Wang, S.Y. (2019) Identify Lysine Neddylation Sites Using Bi-Profile Bayes Feature Extraction via the Chou's 5-Steps Rule and General Pseudo Components. Current Genomics, 20, 592-601.

https://doi.org/10.2174/1389202921666191223154629 
15. Khan, S., Khan, M., Iqbal, N., Hussain, T., Khan, S.A. and Chou, K.C. (2019) A Two-Level Computation Model Based on Deep Learning Algorithm for Identification of piRNA and Their Functions via Chou's 5-Steps Rule. Human Genetics, 19, 756-799. https://doi.org/10.1007/s10989-019-09887-3

16. Khan, Z.U., Ali, F., Khan, I.A., Hussain, Y. and Pi, D. (2019) iRSpot-SPI: Deep Learning-Based Recombination Spots Prediction by Incorporating Secondary Sequence Information Coupled with Physio-Chemical Properties via Chou's 5-Step Rule and Pseudo Components. Chemometrics and Intelligent Laboratory Systems (CHEMOLAB), 189, 169-180. https://doi.org/10.1016/j.chemolab.2019.05.003

17. Lan, J., Liu, J., Liao, C., Merkler, D.J., Han, Q. and Li, J. (2019) A Study for Therapeutic Treatment against Parkinson's Disease via Chou's 5-Steps Rule. Current Topics in Medicinal Chemistry, 19, 2318-2333. https://doi.org/10.2174/1568026619666191019111528

18. Le, N.Q.K. (2019) iN6-methylat (5-Step): Identifying DNA N(6)-methyladenine Sites in Rice Genome Using Continuous Bag of Nucleobases via Chou's 5-Step Rule. Molecular Genetics and Genomics. MGG, 294, 1173-1182. https://doi.org/10.1007/s00438-019-01570-y

19. Le, N.Q.K., Yapp, E.K.Y., Ho, Q.T., Nagasundaram, N., Ou, Y.Y. and Yeh, H.Y. (2019) iEnhancer-5Step: Identifying Enhancers Using Hidden Information of DNA Sequences via Chou's 5-Step Rule and Word Embedding. Analytical Biochemistry, 571, 53-61. https://doi.org/10.1016/j.ab.2019.02.017

20. Le, N.Q.K., Yapp, E.K.Y., Ou, Y.Y. and Yeh, H.Y. (2019) iMotor-CNN: Identifying Molecular Functions of Cytoskeleton Motor Proteins Using 2D Convolutional Neural Network via Chou's 5-Step Rule. Analytical Biochemistry, 575, 17-26. https://doi.org/10.1016/j.ab.2019.03.017

21. Liang, R., Xie, J., Zhang, C., Zhang, M., Huang, H., Huo, H., Cao, X. and Niu, B. (2019) Identifying Cancer Targets Based on Machine Learning Methods via Chou's 5-Steps Rule and General Pseudo Components. Current Topics in Medicnal Chemistry, 19, 2301-2317. https://doi.org/10.2174/1568026619666191016155543

22. Liang, Y. and Zhang, S. (2019) Identifying DNase I Hypersensitive Sites Using Multi-Features Fusion and F-Score Features Selection via Chou’s 5-Steps Rule. Biophysical Chemistry, 253, Article ID: 106227. https://doi.org/10.1016/j.bpc.2019.106227

23. Liu, Z., Dong, W., Jiang, W. and He, Z. (2019) csDMA: An Improved Bioinformatics Tool for Identifying DNA 6 mA Modifications via Chou's 5-Step Rule. Scientific Reports, 9, Article No. 13109. https://doi.org/10.1038/s41598-019-49430-4

24. Malebary, S.J., Rehman, M.S.U. and Khan, Y.D. (2019) iCrotoK-PseAAC: Identify Lysine Crotonylation Sites by Blending Position Relative Statistical Features According to the Chou's 5-Step Rule. PLoS ONE, 14, e0223993. https://doi.org/10.1371/journal.pone.0223993

25. Nazari, I., Tahir, M., Tayari, H. and Chong, K.T. (2019) iN6-Methyl (5-step): Identifying RNA N6-methyladenosine Sites Using Deep Learning Mode via Chou's 5-Step Rules and Chou's General PseKNC. Chemometrics and Intelligent Laboratory Systems (CHEMOLAB), 19, 1-39. https://doi.org/10.1016/j.chemolab.2019.103811

26. Ning, Q., Ma, Z. and Zhao, X. (2019) dForml(KNN)-PseAAC: Detecting Formylation Sites from Protein Sequences Using K-Nearest Neighbor Algorithm via Chou's 5-Step Rule and Pseudo Components. Journal of Theoretical Biology, 470, 43-49. https://doi.org/10.1016/j.jtbi.2019.03.011

27. Tahir, M., Tayara, H. and Chong, K.T. (2019) iDNA6mA (5-step rule): Identification of DNA N6-Methyladenine Sites in the Rice Genome by Intelligent Computational Model via Chou's 5-Step Rule. CHEMOLAB, 189, 96-101. https://doi.org/10.1016/j.chemolab.2019.04.007

28. Wiktorowicz, A., Wit, A., Dziewierz, A., Rzeszutko, L., Dudek, D. and Kleczynski, P. (2019) Calcium Pattern Assessment in Patients with Severe Aortic Stenosis via the Chou's 5-Steps Rule. Current Pharmaceutical Design, 25, 6-31. https://doi.org/10.2174/1381612825666190930101258

29. Yang, L., Lv, Y., Wang, S., Zhang, Q., Pan, Y., Su, D., Lu, Q. and Zuo, Y. (2019) Identifying FL11 Subtype by 
Characterizing Tumor Immune Microenvironment in Prostate Adenocarcinoma via Chou's 5-Steps Rule. Genomics, 112, 1500-1515. https://doi.org/10.1016/j.ygeno.2019.08.021

30. Akbar, S., Rahman, A.U. and Hayat, M. (2020) cACP: Classifying Anticancer Peptides Using Discriminative Intelligent Model via Chou's 5-Step Rules and General Pseudo Components. Chemometrics and Intelligent Laboratory (CHEMOLAB), 196, Article ID: 103912. https://doi.org/10.1016/j.chemolab.2019.103912

31. Akmal, M.A., Hussain, W., Rasool, N., Khan, Y.D., Khan, S.A. and Chou, K.C. (2020) Using Chou's 5-Steps Rule to Predict O-Linked Serine Glycosylation Sites by Blending Position Relative Features and Statistical Moment. IEEE/ ACM Transactions on Computational Biology and Bioinformatics. https://doi.org/10.1109/TCBB.2020.2968441

32. Bouziane, H. and Chouarfia, A. (2020) Use of Chou's 5-Steps Rule to Predict the Subcellular Localization of Gram-Negative and Gram-Positive Bacterial Proteins by Multi-Label Learning Based on Gene Ontology Annotation and Profile Alignment. Journal of Integrative Bioinformatics. https://doi.org/10.1515/jib-2019-0091

33. Charoenkwan, P., Schaduangrat, N., Nantasenamat, C., Piacham, T. and Shoombuatong, W. (2020) iQSP: A Sequence-Based Tool for the Prediction and Analysis of Quorum Sensing Peptides via Chou's 5-Steps Rule and Informative Physicochemical Properties. International Journal of Molecular Sciences, 21, 75. https://doi.org/10.3390/ijms21010075

34. Charoenkwan, P., Schaduangrat, N., Nantasenamat, C., Piacham, T. and Shoombuatong, W. (2020) Correction: Shoombuatong, W., et al. iQSP: A Sequence-Based Tool for the Prediction and Analysis of Quorum Sensing Peptides via Chou's 5-Steps Rule and Informative Physicochemical Properties. International Journal of Molecular Sciences, 21, 75. https://doi.org/10.3390/ijms21072629

35. Chen, Y. and Fan, X. (2020) Use of Chou's 5-Steps Rule to Reveal Active Compound and Mechanism of Shuangshen Pingfei San on Idiopathic Pulmonary Fibrosis. Current Molecular Medicine, 20, 220-230. https://doi.org/10.2174/1566524019666191011160543

36. Dobosz, R., Mucko, J. and Gawinecki, R. (2020) Using Chou's 5-Step Rule to Evaluate the Stability of Tautomers: Susceptibility of 2-[(Phenylimino)-methyl]-cyclohexane-1,3-diones to Tautomerization Based on the Calculated Gibbs Free Energies. Energies, 13, 183. https://doi.org/10.3390/en13010183

37. Du, L., Meng, Q., Jiang, H. and Li, Y. (2020) Using Evolutionary Information and Multi-Label Linear Discriminant Analysis to Predict the Subcellular Location of Multi-Site Bacterial Proteins via Chou's 5-Steps Rule. IEEE Access, 8, 56452-56461. https://doi.org/10.1109/ACCESS.2020.2982160

38. Dutta, A., Dalmia, A., R, A., Singh, K.K. and Anand, A. (2020) Using the Chou's 5-Steps Rule to Predict Splice Junctions with Interpretable Bidirectional Long Short-Term Memory Networks. Computers in Biology and Medicine, 116, Article ID: 103558. https://doi.org/10.1016/j.compbiomed.2019.103558

39. Ju, Z. and Wang, S.Y. (2020) Prediction of Lysine Formylation Sites Using the Composition of k-Spaced Amino Acid Pairs via Chou's 5-Steps Rule and General Pseudo Components. Genomics, 112, 859-866. https://doi.org/10.1016/j.ygeno.2019.05.027

40. Kabir, M., Ahmad, S., Iqbal, M. and Hayat, M. (2020) iNR-2L: A Two-Level Sequence-Based Predictor Developed via Chou's 5-Steps Rule and General PseAAC for Identifying Nuclear Receptors and Their Families. Genomics, 112, 276-285. https://doi.org/10.1016/j.ygeno.2019.02.006

41. Khan, Y.D., Amin, N., Hussain, W., Rasool, N., Khan, S.A. and Chou, K.C. (2020) iProtease-PseAAC(2L): A Two-Layer Predictor for Identifying Proteases and Their Types Using Chou's 5-Step-Rule and General PseAAC. Analytical Biochemistry, 588, Article ID: 113477. https://doi.org/10.1016/j.ab.2019.113477

42. Lin, W., Xiao, X., Qiu, W. and Chou, K.C. (2020) Use Chou's 5-Steps Rule to Predict Remote Homology Proteins by Merging Grey Incidence Analysis and Domain Similarity Analysis. Natural Science, 12, 181-198. https://doi.org/10.4236/ns.2020.123016 
43. Lu, W., Song, Z., Ding, Y., Wu, H., Cao, Y. and Zhang, Y.L. (2020) Use Chou's 5-Step Rule to Predict DNA-Binding Proteins with Evolutionary Information. BioMed Research International, 2020, Article ID: 6984045. https://doi.org/10.1155/2020/6984045

44. Nguyen, D., Ho-Quang, T., Nguyen Quoc Khanh, L., Dinh-Phan, V. and Ou, Y.Y. (2020) Use Chou's 5-Steps Rule with Different Word Embedding Types to Boost Performance of Electron Transport Protein Prediction Model. IEEE/ACM Transactions on Computational Biology and Bioinformatics. https://doi.org/10.1109/TCBB.2020.3010975

45. Pandey, R.P., Kumar, S., Ahmad, S., Vibhuti, A., Raj, V.S., Verma, A.K., Sharma, P. and Leal, E. (2020) Use Chou's 5-Steps Rule to Evaluate Protective Efficacy Induced by Antigenic Proteins of Mycobacterium tuberculosis Encapsulated in Chitosan Nanoparticles. Life Sciences, 256, Article ID: 117961. https://doi.org/10.1016/j.lfs.2020.117961

46. Roy, T. and Bhattacharjee, P. (2020) A LabVIEW-Based Real-Time Modeling Approach via Chou's 5-Steps Rule for Detection of Abnormalities in Cancer Cells. Gene Reports, 20, Article ID: 100788. https://doi.org/10.1016/j.genrep.2020.100788

47. So, C. and Yang, B. (2020) Use Chou's 5-Step Rule to Classify Protein Modification Sites with Neural Network. Scientific Programming, 2020, Article ID: 8894633. https://doi.org/10.1155/2020/8894633

48. Song, C. and Yang, B. (2020) Use Chou's 5-Step Rule to Classify Protein Modification Sites with Neural Network. Scientific Programming, 2020, Article ID: 8894633. https://doi.org/10.1155/2020/8894633

49. Vishnoi, S., Garg, P. and Arora, P. (2020) Physicochemical n-Grams Tool: A Tool for Protein Physicochemical Descriptor Generation via Chou's 5-Step Rule. Chemical Biology \& Drug Design, 95, 79-86. https://doi.org/10.1111/cbdd.13617

50. Vundavilli, H., Datta, A., Sima, C., Hua, J., Lopes, R. and Bittner, M. (2020) Using Chou's 5-Steps Rule to Model Feedback in Lung Cancer. IEEE Journal of Biomedical and Health Informatics, 21, 1-24. https://doi.org/10.1109/JBHI.2019.2958042

51. Yang, L., Lv, Y., Wang, S., Zhang, Q., Pan, Y., Su, D., Lu, Q. and Zuo, Y. (2020) Identifying FL11 Subtype by Characterizing Tumor Immune Microenvironment in Prostate Adenocarcinoma via Chou's 5-Steps Rule. Genomics, 112, 1500-1515. https://doi.org/10.1016/j.ygeno.2019.08.021

52. Zhang, S. and Xue, T. (2020) Use Chou's 5-Steps Rule to Identify DNase I Hypersensitive Sites via Dinucleotide Property Matrix and Extreme Gradient Boosting. Molecular Genetics and Genomics. MGG, 295, 1431-1442. https://doi.org/10.1007/s00438-020-01711-8 